\title{
Intraoperative Radiotherapie des konservativ operierten Mammakarzinoms
}

\author{
Gesprächsleitung: Felix Sedlmayer ${ }^{\mathrm{a}}$
}

\author{
Teilnehmer: Michael Eble ${ }^{b} \quad$ Wolfgang Harms $^{c} \quad$ Marie-Luise Sautter-Bihl ${ }^{d} \quad$ Árpád Sztankay \\ Frederik Wenz ${ }^{f} \quad$ Normann Willich ${ }^{g}$
}

\author{
a Universitätsklinik für Radiotherapie und Radio-Onkologie, Landeskrankenhaus Salzburg/Universitätsklinikum der Paracelsus \\ Medizinischen Privatuniversität (PMU), Salzburg, Österreich \\ ${ }^{b}$ Klinik für Strahlentherapie, Universitätsklinikum der RWTH Aachen, Deutschland \\ ${ }^{\mathrm{c}}$ Radioonkologie, St. Claraspital, Basel, Schweiz \\ ${ }^{\mathrm{d}}$ Klinik für Strahlentherapie, Städtisches Klinikum Karlsruhe, Deutschland \\ e Universitätsklinik für Strahlentherapie und Radioonkologie, Medizinische Universität Innsbruck, Österreich \\ f Universitätsklinik für Strahlentherapie und Radioonkologie, Universitätsmedizin Mannheim, Deutschland \\ ${ }^{g}$ Klinik und Poliklinik für Strahlentherapie - Radioonkologie, Universitätsklinikum Münster, Deutschland
}

Die Bezeichnung "IORT» (intraoperative Radiotherapie) wird derzeit für vier verschiedene Techniken verwendet: IORT mittels Elektronen am Linearbeschleuniger (IOERT), mittels Orthovoltbestrahlung (Intrabeam ${ }^{\circledR}$, Carl Zeiss Meditec AG, Jena, Deutschland), als perioperative interstitielle Multikatheter-Brachytherapie oder als endokavitäre Brachytherapie (MammoSite ${ }^{\circledR}$, Hologic Deutschland GmbH, Frankfurt/M., Deutschland). Nur die beiden ersteren Methoden sind Intraoperativbestrahlungen im strengen Wortsinn. Diese Verfahren weisen untereinander enorme dosimetrische Unterschiede auf, so dass klinische Resultate streng in Abhängigkeit von der verwendeten Methode zu werten sind. Bis dato sind die meisten publizierten Lokalrezidivraten auffallend niedrig.

Die bislang beste Datenlage besteht für die Boost-IOERT, für die Erfahrungen einer gepoolten Europäischen Analyse an über 1100 Patientinnen vorliegen. Nach über 5-jähriger medianer Nachbeobachtungszeit werden jährliche InBrust-Rezidivraten von lediglich $0,2 \%$ berichtet.

Frage 1: Wie bewerten Sie die unterschiedlichen Verfahren für eine IORT?

Eble: Die unterschiedlichen Verfahren der IORT können bisher nur anhand strahlenphysikalischer Gegebenheiten, logistischer und methodischer Verfahrensweisen sowie bei unterschiedlicher Strahlqualität anhand möglicher strahlbiologisch experimenteller Implikationen bewertet werden. Verwertbare klinische Daten liegen nicht vor. Da letztere für die klinische Anwendung entscheidend sind, kommen aktuell alle Verfahren zum Einsatz.

Wenz: Die vorhandenen Techniken - IORT mit Elektronen oder Photonen - haben jeweils spezifische Vor- und Nachteile bezüglich OP-Technik, Strahlenschutz und Kosten. Eines haben die Verfahren jedoch gemeinsam, sie erfordern ein hohes Maß an Expertise und an interdisziplinärer Abstimmung.

Harms: Intraoperative Bestrahlungen mit Elektronen oder mit niederenergetischen Röntgenstrahlen haben im Vergleich zu Verfahren, bei denen in der Regel nach intraoperativer Applikatoreinlage (Multikatheter oder endokavitäre Brachytherapie) die Bestrahlung postoperativ erfolgt, den Vorteil, dass die Bestrahlung unmittelbar während des operativen Eingriffes mit direkter Bestrahlung des Tumorbettes erfolgen kann. Hierdurch ergeben sich neben zeitlichen und technischen, insbesondere auch psychologische Vorteile für die Patientinnen, da die Bestrahlung mit Beendigung der Operation bereits durchgeführt wurde. Auf der anderen Seite stellen diese Methoden jedoch sehr hohe Anforderungen an die

\section{KARGER}

Fax +497614520714

Information@Karger.de

www.karger.com (c) 2010 S. Karger GmbH, Freiburg

Accessible online at:

www.karger.com/brc
Univ.-Prof. Dr. Felix Sedlmayer

Univ-Klinik für Radiotherapie und Radio-Onkologie, Landeskrankenhaus Salzburg Universitätsklinikum der Paracelsus Medizinischen Privatuniversität (PMU) Salzbur Müllner Hauptstr.48, 5020 Salzburg, Österreich

Tel. +43662 44823904

f.sedlmayer@salk.at 
Qualität der intraoperativen Schnellschnittdiagnostik, bzw. haben den Nachteil, dass zum Zeitpunkt der Bestrahlung noch kein endgültiger pathohistologischer Bericht vorliegt.

Sautter-Bihl: Grundsätzlich ist zu unterscheiden, ob das Verfahren als vorweggenommener Boost oder als alleinige Teilbrustbestrahlung zum Einsatz kommt. Von einer BoostBestrahlung mit ca. 10-16 Gy profitiert nach den Ergebnissen der randomisierten EORTC-Studie jede Alters- und Risikogruppe. Nachdem die Boost-IORT von einer GanzbrustRadiotherapie (RT) ergänzt wird, nimmt dieses Konzept Rücksicht auf das unterschiedlich hohe Risiko einer okkulten Tumorzellaussaat außerhalb des Indexquadranten und eignet sich somit in jeder Risikokonstellation einer brusterhaltenden Therapie. Die Rationale der alleinigen «Volldosis»-IORT mit einer Einzeldosis bis zu 24 Gy basiert auf der Beobachtung, dass Tumorrezidive überwiegend im Bereich des ehemaligen Tumorbettes beschrieben werden, weshalb das Potential eines Ersatzes der Ganzbrust-RT bei Niedrig-Risiko-Patientinnen untersucht wird. Wiewohl der offensichtliche Vorteil in einer substantiellen Verkürzung der Gesamtbehandlungsdauer liegt, birgt dieses Vorgehen das Risiko eines «geographic miss» sowohl in der Tumorbettperipherie als auch außerhalb des Indexquadranten, wo keine Ganzbrust-RT mehr wirksam wird.

Willich: Die Tumorwirksamkeit ist etwa gleich bei IORT mit Brachytherapie, Intrabeam, Mammosite und Elektronen. Die Homogenität der Dosisverteilung ist am besten bei Elektronenbestrahlung. Das Risiko für Hautnebenwirkungen ist am geringsten bei Elektronen-IORT. Die niedrigsten Lokalrezidivraten bei zugleich bester Kosmetik erreicht man wahrscheinlich durch IORT mit Elektronen.

\section{Frage 2: Worin liegt der potentielle Vorteil einer intraoperativen Bestrahlung?}

Eble: Der potentielle Vorteil der IORT liegt in der hohen Treffsicherheit - weniger geographic miss des tumortragenden Risikogebietes. Damit korreliert die verminderte DosisVolumen-Belastung des mitbetroffenden Normalgewebes und das verbesserte funktionelle bzw. kosmetische Ergebnis.

Wenz: Der große Vorteil der IORT beim Mammakarzinom ist die Vermeidung eines geographic miss sowie die frühestmögliche Applikation der Radiotherapie. Ein geographic miss, d.h. eine mögliche, teilweise Fehllokalisation der Boostbestrahlung kann durch derzeitige Techniken bei der perkutanen Boostbestrahlung nicht ausgeschlossen werden.

Harms: Die IORT ermöglicht im Gegensatz zu einer mehrwöchigen externen Bestrahlung eine einmalige oder akzelerierte Bestrahlung des Tumorbettes. Durch die direkte intraoperative Bestrahlung kann eine bessere Lokalisation des Tumor- bettes erfolgen. Zusätzlich erfolgt die Bestrahlung zum frühestmöglichen und somit effektivsten Zeitpunkt. Ebenso müssen positive Effekte auf das Mikromilieu diskutiert werden (Hypothese: verminderte Ausschüttung von Zytokinen und Wachstumsfaktoren).

Sztankay: Der potentielle Vorteil einer IORT besteht darin, dass unmittelbar nach der Tumorresektion im Bereich des ehemaligen Tumorsitzes, wo die Gefahr und Wahrscheinlichkeit eines Tumorrezidivs am höchsten ist, eine hohe und biologisch hocheffektive Dosisapplikation im Sinne einer lokalen Dosisaufsättigung erfolgen kann. Die Einmalbestrahlung kann unter operativen Bedingungen mit hoher Konformität durchgeführt werden, normales Gewebe und Risikoorgane können vom Bestrahlungsvolumen exkludiert werden. Durch die intraoperative Verabreichung einer hohen Einzeldosis kann die Gesamtdosis der anschließenden perkutanen Radiotherapie und somit auch deren Spätfolgen entsprechend reduziert werden.

Willich: Als Boostbestrahlung liegt der Vorteil in der Verkürzung der gesamten perkutanen Bestrahlungsserie um ca. 1,5 Wochen. Weitere potentielle Vorteile sind: hohe lokale Wirksamkeit mit exzellent geringen lokalen Rezidivraten und bei den verschiedenen Verfahren unterschiedlich gute, z. T. durchgehend hervorragend gute kosmetische Ergebnisse.

\section{Frage 3: Wie ist die intraoperative Applikation einer einmalig hohen Strahlendosis beim Mammakarzinom aus biologischer Sicht zu werten?}

Eble: Die einmalige hohe Strahlendosis belastet das Tumorbett und damit den Brustdrüsenkörper und kann dort aufgrund der verminderten Reparatur des genetischen Strahlenschadens die chronische Morbidität erhöhen. Die klinische und radiologische Bewertung zeigt jedoch, dass das Risiko einer relevanten Fettnekrose im Strahlgebiet nicht erhöht ist. Die kritische Belastung der Rippen oder der Lunge wird durch eine angepasste Wahl der Elektronenenergie oder durch die Einlage eines Strahlungsabsorbers abgefangen.

Wenz: Eine exakte Quantifizierung des Effektes kann mit den derzeitigen Modellrechnungen nicht erfolgen. Man kann jedoch abschätzen, dass IORT-Dosen von 10-20 Gy einer fraktionierten Dosis von mehr als 30 Gy entsprechen. Eine Bestrahlung schon während der Operation vermindert das Risiko, dass sich Tumorzellen zwischen der Operation und dem Beginn der Bestrahlung bereits vermehren.

Harms: Auf der Basis klassischer radiobiologischer Modelle muss bei der Verwendung hoher Einzeldosen prinzipiell mit einer erhöhten Rate an Spätnebenwirkungen am Normalgewebe wie Teleangiektasien, Fibrosen oder Fettgewebsnekro- 
sen und möglicherweise suboptimalen kosmetischen Ergebnissen gerechnet werden. Andererseits ermöglicht gerade die intraoperative Bestrahlung, direkt das Tumorbett zu bestrahlen und somit die Haut zu schonen. Zusätzlich weisen neuere klinische Daten auf eine wahrscheinlich erhöhte intrinsische Strahlenempfindlichkeit von Mammakarzinomen gegenüber erhöhten Einzeldosen hin. Falls sich diese Beobachtungen bestätigen sollten, wäre mit einer erhöhten Wirksamkeit hoher Einzeldosen zu rechnen.

Sztankay: Die Applikation einer hohen Einzeldosis auf die Resektionslage des Tumors im Rahmen einer brusterhaltenden Operation ermöglicht eine präzise und hochkonformale Dosiseskalation im Bereich der höchsten Wahrscheinlichkeit der Tumorwiederentstehung. Die Wirksamkeit einer intraoperativen Bestrahlung im Hinblick auf die lokale Tumorkontrolle wurde durch eine Vergleichsstudie (IORT vs. perkutaner Boost) an einer größeren Patientenzahl $(\mathrm{n}=378)$ an der medizinischen Universität Salzburg untersucht und belegt (Reitsamer et al., Strahlenther Onkol 2004;180:38-44). Über die hohe Effektivität der IORT berichte auch die Studiengruppe aus Mailand (Veronesi et al., Ann Surg 2005;242:101106). Die Anwendung der IORT mittels Elektronen mit anschließender adjuvanter perkutaner Radiotherapie ist bei Verfügbarkeit dieser Technik weitgehend zu befürworten.

Willich: Eine Boostdosis in der Größenordnung einer Einzeldosis von ca. 9 Gy ersetzt hinsichtlich der Tumorwirkung etwa 18-20 Gy einer normofraktionierten Bestrahlung und ist damit eine hochwirksame Dosis.

Sautter-Bihl: Im Vergleich zu anderen Tumoren wie beispielsweise Plattenepithelkarzinomen (SCC) scheinen Mammakarzinomzellen eine verstärkte Sensibilität gegenüber hohen Einzeldosen aufzuweisen. Zur Effektbeschreibung unterschiedlicher Strahlendosen wird meist das sogenannte linearquadratische Modell verwendet, welches einen Zusammenhang zwischen Einzeldosis, Gesamtdosis und biologisch effektiver Dosis herstellt, und zwar basierend auf einer «Gewebskonstante», dem sogenannten $\alpha / \beta$-Wert. Für Mammakarzinome wurde experimentell ein Wert von 4 ermittelt, der sich in kanadischen und britischen Studien zur Hypofraktionierung bestätigte - anstatt 10 für die meisten Plattenepithelkarzinome. Diese niedrigere Ratio bedeutet eine höhere Empfindlichkeit gegenüber einer höheren Einzeldosis - ein starkes Argument für die intraoperative Applikation einer hohen Einzeldosis. Gemäß diesem biologischen Modell entspricht somit die Wirksamkeit eines IORT-Boosts von 10 Gy einem herkömmlichen Boost von 24 Gy in 2-Gy-Einzelfraktionierung. Allerdings wurde dieses Modell nur für Einzeldosen bis 15 Gy geprüft, seine Gültigkeit bei alleiniger IORT mit 24 Gy (wie im ELIOT-Trial verwendet) ist völlig offen, sowohl hinsichtlich zu erwartender Effekte am Tumor als auch an Normalgeweben (Spätreaktionen).

\section{Frage 4: Ist die alleinige IORT eine Option bei Patientinnen mit niedrigem Lokalrezidivrisiko?}

Eble: Basierend auf einer detaillierten Lokalrezidivanalyse der Phase-III-Milan-Studie zur ergänzenden Radiotherapie nach brusterhaltender Operation wird das progressionsfreie Überleben eindeutig vom Lokalrezidiv im betroffenen Quadranten bestimmt. Weitere Analysen, im wesentlichen aus dem Bereich der Brachytherapie zeigen, dass bei reduzierter lokaler Tumorlast, kombiniert mit dem Fehlen von Risikofaktoren einer häufigen lokoregionalen Tumorpropagation, eine hohe lokoregionale Tumorkontrolle erreicht werden kann. Die alleinige IORT wird eine Option beim kleinen Mammakarzinom darstellen. Der notwendige Beleg aus Phase-IIIStudien steht noch aus.

Wenz: Dies ist derzeit sicherlich eine der wichtigsten Fragen in der Behandlung des Mammakarzinoms. Zum jetzigen Zeitpunkt ist die alleinige IORT nur innerhalb von Studien eine Option, da noch keine reifen Daten der laufenden Studien mit ausreichender Nachsorgezeit vorliegen. Erste Daten werden in diesem Jahr erwartet, eine Umsetzung in die klinische Praxis wird jedoch frühestens in ca. 2 Jahren erwartet.

Harms: Im Prinzip und vom theoretischen Ansatz her ja, allerdings sollte außerhalb von Studien eine IORT nur als Boostbestrahlung des Tumorbettes in Kombination mit einer mehrwöchigen externen Bestrahlung erfolgen. Die Wertigkeit einer alleinigen Teilbrustbestrahlung mittels IORT wird derzeit in prospektiven Studien untersucht und muss bis zum Vorliegen belastbarer Daten als experimentell eingestuft werden.

Sztankay: Eine alleinige IORT bei Patientinnen mit niedrigem Lokalrezidivrisiko des Mammakarzinoms kann derzeit nicht außerhalb von Studien empfohlen werden. Vorläufige Kurzzeitergebnisse der Arbeitsgruppe aus Mailand zeigen bei mehr als 500 Patientinnen eine sehr gute lokale Tumorkontrolle und niedrige Lokalrezidivrate nach alleiniger IORT. Die Evidenz der Anwendbarkeit einer alleinigen IORT mit Elektronen bei Patientinnen mit einem niedrigem Lokalrezidivrisiko muss noch durch die Langzeitergebnisse der randomisierten Studie von Mailand bestätigt werden.

Willich: Die alleinige IORT als Alternative zu einer Bestrahlung der ganzen Brust scheint bei adäquater Auswahl der Patientinnen (ältere Patientinnen mit niedrigem Risiko) nach den bisherigen Erfahrungen günstige Ergebnisse liefern zu können. Aber: Bisher wurde dieses Verfahren nur in kleinen Studien untersucht, es liegen nicht genügend Langzeitergebnisse vor, das Risiko schlechter kosmetischer Ergebnisse besteht und die Gleichwertigkeit zur Ganzbrustbestrahlung ist bisher nicht erwiesen. Derzeit ist die alleinige IORT als experimentell einzuschätzen. 


\section{Teilnehmer}

Prof. Dr. Michael Eble

Klinik für Strahlentherapie

Universitätsklinikum der RWTH Aachen

Pauwelsstr. 30, 52074 Aachen, Deutschland

Tel. +492418089261

meble@ukaachen.de

Prof. Dr. Wolfgang Harms

Radioonkologie

St. Claraspital

Kleinriehenstr. 30, 4058 Basel, Schweiz

Tel. +41616858585

wolfgang.harms@claraspital.ch

Prof. Dr. Marie-Luise Sautter-Bihl

Klinik für Strahlentherapie

Städtisches Klinikum Karlsruhe

Moltkestr. 90, 76131 Karlsruhe, Deutschland

Tel. +49721974 4001

marie-luise.sautter-bihl@klinikum-karlsruhe.de
OA Dr. Árpád Sztankay

Univ.-Klinik für Strahlentherapie-Radioonkologie

Medizinische Universität Innsbruck

Anichstr. 35,6020 Innsbruck, Österreich

Tel. +43 512 504-22800 od. -22799

arpad.sztankay@uki.at

Prof. Dr. med. Frederik Wenz

Universitätsklinik für Strahlentherapie und Radioonkologie

Universitätsmedizin Mannheim

Theodor-Kutzer-Ufer 1-3, 68135 Mannheim, Deutschland

Tel. +496213834960 od. 1540

frederik.wenz@umm.de

Prof. Dr. Normann Willich

Klinik und Poliklinik für Strahlentherapie - Radioonkologie Universitätsklinikum Münster

Albert-Schweitzer-Straße 33, 48129 Münster, Deutschland

Tel. +492518347384

normann-willich@ukmuenster.de 